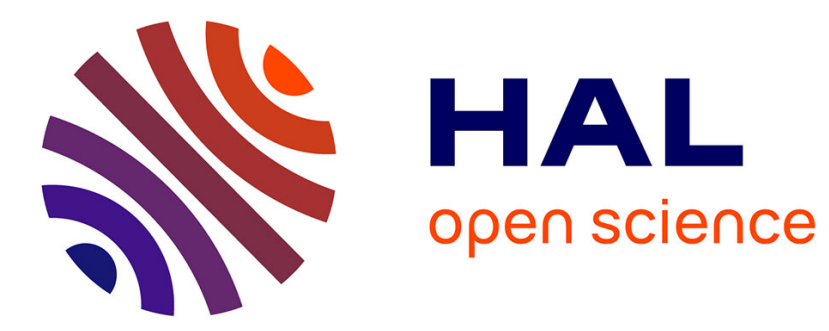

\title{
Morphogenèse à partir du stade juvénile de Vitis vinifera $L$. issu de graine ou de culture in vitro
}

René Nozeran, Serge Grenan, Paul Truel, Jean-Michel Favre

\section{To cite this version:}

René Nozeran, Serge Grenan, Paul Truel, Jean-Michel Favre. Morphogenèse à partir du stade juvénile de Vitis vinifera L. issu de graine ou de culture in vitro. Agronomie, 1983, 3 (7), pp.681-684. hal00884559

\section{HAL Id: hal-00884559 \\ https://hal.science/hal-00884559}

Submitted on 1 Jan 1983

HAL is a multi-disciplinary open access archive for the deposit and dissemination of scientific research documents, whether they are published or not. The documents may come from teaching and research institutions in France or abroad, or from public or private research centers.
L'archive ouverte pluridisciplinaire HAL, est destinée au dépôt et à la diffusion de documents scientifiques de niveau recherche, publiés ou non, émanant des établissements d'enseignement et de recherche français ou étrangers, des laboratoires publics ou privés. 


\title{
Morphogenèse à partir du stade juvénile de Vitis vinifera L. issu de graine ou de culture in vitro
}

\author{
René NOZERAN, Serge GRENAN*, Paul TRUEL ** \& Jean-Michel FAVRE *** \\ Université Paris-sud, Laboratoire de Morphologie végétale expérimentale, associé au CNRS, F91405 Orsay \\ * Association Nationale Technique pour l'Amélioration de la Viticulture (ANTAV), Domaine de l'Espiguette, \\ F 30240 Le Grau-du-Roi \\ ** I.N.R.A., Station de Recherches de Viticulture, Domaine de Vassal, Centre de Recherches de Montpellier, \\ F 34340 Marseillan \\ *** Université de Nancy I, Laboratoire de Morphologie végétale, F 54506 Vandceuvre Cedex
}

RÉSUMÉ

Des observations concordantes ont été faites sur des individus de Vitis vinifera $\mathrm{L}$. issus de multiplications in vitro et sur d'autres provenant de semences. Elles laissent penser que les différences de morphogenèse de ces plantes par rapport aux individus d'origine sont le fait d'un fonctionnement du matériel héréditairc en phase juvénilc. Celui-ci peut être perpétué du fait de contraintes écologiques (peut-ĉtre la culture in vitro, sûrement le climat) associées à des pratiques agronomiques (taille). Des éléments sont ainsi fournis pour améliorer la gestion agronomique de Vitis vinifera.

Mots clés additionnels : Vigne, gradients morphogénétiques, fonctionnement juvénile, variants.

Identical observations were made on Vitis vinifera $\mathbf{L}$. individuals produced either by in vitro multiplication or from seed. The morphogenetic differences between these plants and their plants of origin, are the result of a malfunction of their genetic material stabilized in a juvenile state. This state can be perpetuated by ccological constraints (in vitro culture possibly, climate certainly, and ccological constraints associated with agronomical techniques such as pruning. Information is thus given on better management of Vitis vinifera $\mathrm{L}$.

Additional key words : Grapevine, morphogenetic gradients, juvenility, variations.

\section{INTRODUCTION}

La multiplication in vitro associéc à des traitements par thermothérapic a conduit à des résultats dont certains sont utilisés dans la pratique agronomique. C'est ainsi qu'à partir d'individus de Vitis rupestris Scheele très sévèrement infestés par le virus du court-noué, on a pu obtenir des clones sains (GALZY, 1961) qui ont servi de point de départ à une cxploitation au vignoble (VALAT et al., 1973, 1979, 1981). Mais les résultats sont moins encourageants pour les divers clones de $V$. vinifera $\mathrm{L}$. Bien que la technique ait permis l'élimination de virus, les plants issus de ces pratiques peuvent montrer, dans les conditions du vignoble, des performances de production nettement inférieures à celles des témoins. Cette baisse de productivité, celle-ci pouvant être quasiment nulle, est concomitante de l'apparition de types de morphogenèse, toujours les mêmes, ne se manifestant pas sur l'individu de départ. Il s'agit de la forme plus ou moins échancrée et de la pubescence des feuilles, ainsi que de la pigmentation des jeunes rameaux (BRANAS, 1974;
VALAT \& MUR, 1976 ; VAlat et al., 1979 ; VALAT et al., 1981), (fig. 1).

L'analyse expérimentale de ce phénomène conduit à penser que ce mode de fonctionnement apparemment particulier est la conséquence durable des processus de rajeunissement consécutifs à la culture in vitro (accompagnés ou non de thermothérapie). On sait, en effet, qu'en les plaçant dans des conditions écologiques de ce type, on peut obtenir le rajeunissement de diverses plantes, l'acquisition des caractéristiques de celui-ci progressant au fil des repiquages. La miniaturisation du méristème édificateur de l'axe accompagne ce phénomène ; il en est, peut-être, au moins un des éléments de son déterminisme (NOZERAN \& BANCILHON, 1972; NOZERAN et al., 1977; NOZERAN, 1980 ; Nozeran et al., 1982 ; WATElET-GONOD \& FAVRE, 1981).

Chez $V$. vinifera, les individus ainsi obtenus, y compris ceux issus des premières multiplications in vitro, conservent longtemps certains traits juvéniles lorsqu'ils sont plantés au vignoble. Ceci ne va pas sans inconvénientš, tant du fait de 

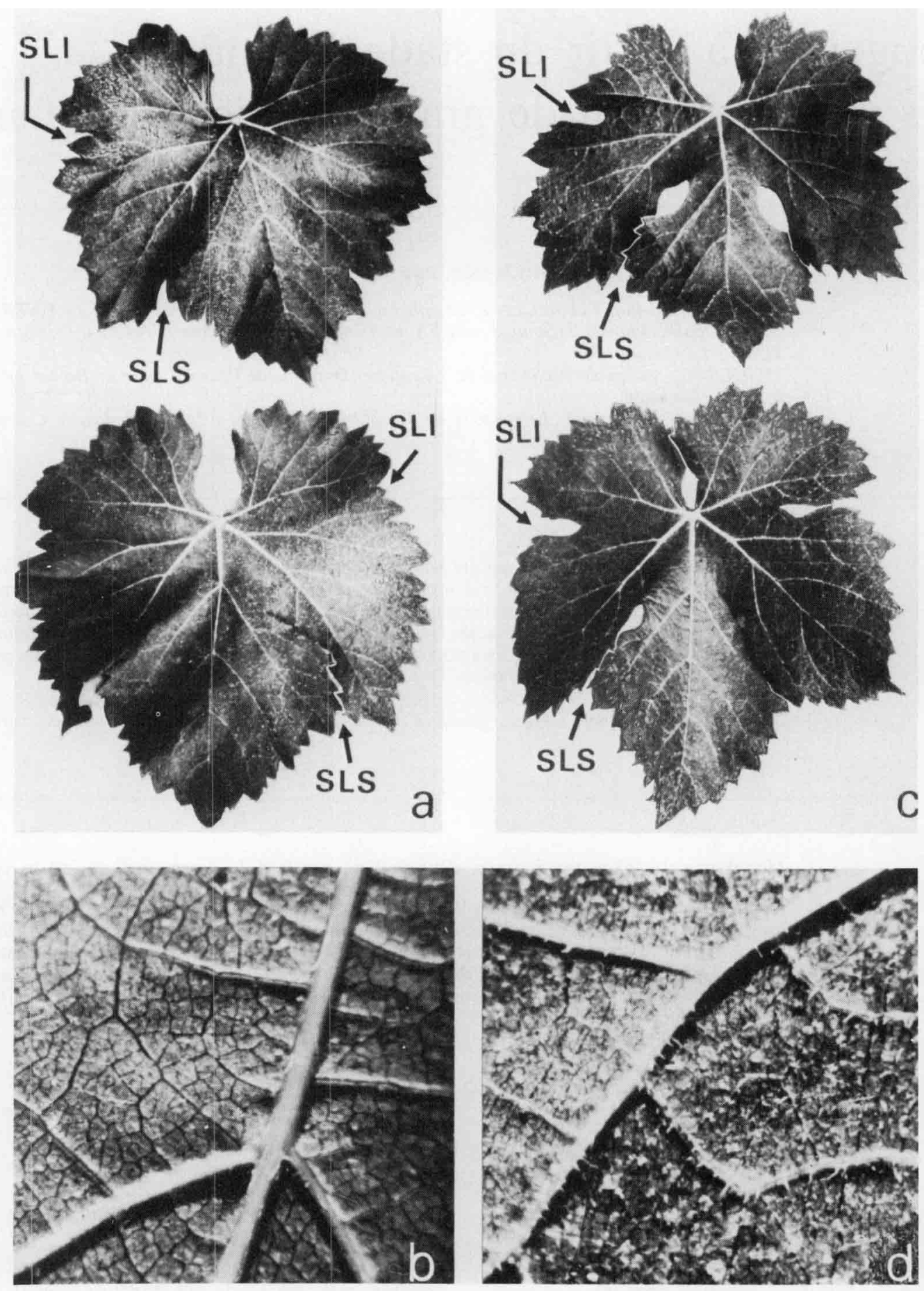

Figure 1

Comparaison des feuilles adultes et juvéniles de la variété "Grenache N."

Feuilles adultes avant passage en culture in vitro.

a) Feuilles à sinus latéraux peu prononcés.

b) Face inférieure du limbe glabre.

Feuilles invéniles après passage en culture in vitro.

c) Feuilles à sinus latéraux très prononcés.

d) Face inférieure du limbe pubescente (nombreux poils dressés).

S.L.S. : Sinus latéraux supérieurs.

S.L.I. : Sinus latéraux inférieurs.

Comparison of the adult and juvenile leaves of "Grenache N.". Adult leaves before in vitro culture.

a) Shallow lateral sinuses of the leaves.

b) Hairless lower surface of the leaf blade.

Juvenile leaves after in vitro culture.

c) Deep lateral sinuses of the leaves.

d) Pubescent lower surface of the leaf blade (many short, erect hairs).

S.L.S. : Upper lateral sinuses.

S.L.I. : Lower lateral sinuses. 
la réduction parfois très importante de la productivité, ce qui est bien évidemment décisif pour l'agronome, que de l'apparition des traits morphologiques nouveaux déjà mentionnés qui rendent inutilisables ces clones du fait de la législation française actuelle relative au classement des « cépages ».

\section{OBSERVATIONS ET EXPÉRIENCES}

L'analyse du déterminisme de ce comportement singulier a été conduite par l'un d'entre nous (GRENAN, 1982) au laboratoire de l'ANTAV. On a pu montrer que la perpétuation d'un tel type de morphogenèse était sous la dépendance de 2 facteurs: l'un climatique, l'autre lié aux pratiques culturales, tous 2 concourant à l'élimination des portions terminales des rameaux. On sait, en effet, qu'à l'automne, on constate la mort et la chute de ces dernières. En outre, la taille pratiquée par le viticulteur ne laisse subsister que la partie basale du sarment et ne conserve sur celui-ci (au moins dans le midi méditerranéen) que 2 bourgeons.

Or, si par un artifice cultural on évite ces phénomènes, par exemple en plaçant les individus de «Grenache N. » en conditions favorables à une croissance continue, on s'aperçoit que les tiges obtenues finissent par porter des feuilles dont les caractéristiques morphologiques reproduisent celles qui servent de critères ampélographiques de reconnaissance de la variété qui a servi de point de départ (feuilles glabres, simplement lobées). Le bouturage de cette portion terminale conduit à un individu conservant ces traits.

De même, si au lieu de pratiquer la taille courte habituelle, on laisse subsister chez le «Muscat à petits grains B. » un nombre d'yeux plus important (12), on s'aperçoit que les supérieurs donnent naissance à des rameaux porteurs de fructifications alors que les inférieurs, les seuls laissés par la taille classique, n'en portent pas.

L'interprétation la plus simple de ces faits est que, partant d'un type de fonctionnement juvénile comparable à celui d'un individu issu de graine mais obtenu par la culture in vitro, le déploiement morphogénétique jusqu'à l'état adulte est freiné par des contingences écologiques (mort et chute des sommets des rameaux) et agronomique (taille). Chaque croissance au printemps se faisant, de ce fait, à partir des bourgeons de la base du rameau, dont on a pu montrer pour d'autres espèces qu'elle ést le siège de phénomènes de rajeunissement (NOZERAN, 1978), il en résulte une perpétuation artificielle des caractéristiques juvéniles au fil des ans.

Nous ne pouvons pas affirmer que la culture in vitro ne conduit pas à une situation d'activité du matériel héréditaire se traduisant au vignoble, pour l'individu qui en est issu, par une prolongation de fonctionnements comparables à ceux du jeune individu issu d'une graine. Une perpétuation de cet état, réversible à terme, constituerait une illustration de la situation «variant» au sens donné au terme lorsque son utilisation a été proposée (NOZERAN \& BANCILHON, 1972). Mais même si cela est le cas, il n'en reste pas moins vrai que toutes les informations disponibles montrent qu'il s'agit là de modalités de morphogenèse, c'est-à-dire de modalités de fonctionnement du matériel héréditaire qui, dans tous les cas mais plus ou moins rapidement, pourront être dépassées.

Cependant, la validité de cette explication est subordonnée à l'existence de phénomènes comparables lorsqu'on s'adresse à des individus issus de semences. De ce point de vue, les observations réalisées sur une nouvelle variété de raisin de table, «Danam B. » issue du croisement « Dabouki B. » $x$ «Muscat de Hambourg N. », se sont révélées particulièrement instructives (GRENAN \& TRUEL, 1983). Partant du plant hybride par multiplication végétative (bouturage), on obtient, en effet, une $1^{\text {re }}$ descendance d'individus possédant tous des rameaux anthocyaniques dont les feuilles sont profondément découpées, ont des nervures et pétioles eux-mêmes vivement colorés et présentent une abondante pubescence sur leur face inférieure. Toujours par multiplication végétative (bouturage et greffage), on peut observer, par la suite, l'apparition de souches, de plus en plus nombreuses au fil des années, exhibant des caractéristiques nouvelles: feuilles moins découpées, disparition quasi totale de la coloration anthocyanique des rameaux, des pétioles et des nervures, diminution de la pubescence des faces inférieures des feuilles. Tout se passe comme si, au cours du temps, à la suite des traitements agronomiques subis (on pourrait dire malgré ces traitements), certains des individus issus de multiplication végétative avaient eu la possibilité de franchir les étapes de leur morphogenèse normale et d'accéder à l'état adulte.

Dans le même esprit, il est intéressant de constater que, lorsqu'ils se développent, les gourmands nés de la base de la souche (même si celle-ci a un fonctionnement adulte), présentent des caractéristiques de la plante issue de graine. Or, on sait maintenant que, chez les plantes ligneuses, les rameaux issus de la région proche du système racinaire et dont les méristèmes subissent généralement une diapause pouvant être longue, présentent par rapport au pied-mère des caractères qu'ils ont en commun avec des individus issus de la semence (NOZERAN et al., 1977 ; NozERAN et al., 1982).

Ces observations portant sur les individus issus de graines jointes à des faits signalés incidemment par d'autres chercheurs recoupent celles qui ont êté réalisées sur les individus issus de multiplication in vitro. Ces derniers ne peuvent donc être utilisés en toute sécurité dans la pratique agronomique que dans la mesure où ils seront soumis à des traitements permettant de maitriser le passage d'un mode de fonctionnement juvénile à un mode de fonctionnement adulte.

Parmi ceux-ci, on peut compter, bien entendu, une modulation des processus de taille des individus au vignoble. Mais, avant la première installation dans le site de culture, ne pourrait-on pas faire «vieillir " la plante après sa sortie de tube en appliquant la technique que HUGLIN \& JULLIARD (1964) utilisent pour les plantes de semis et dont WAGNER \& BRONNER (1974) ont précisé l'impact. Après un an de végétation en culture hydroponique en serre, les plantes issues de graines présentent des bourgeons avec inflorescences, la fertilité de ceux-ci dépendant, en particulier, de leur rang sur l'axe. WAGNER \& BRONNER (1974) ont déterminé le niveau de la taille à appliquer à ces individus afin d'obtenir à la fois une bonne expression de la fertilité et une production suffisante d'yeux pour le greffage ou le bouturage. Celui-ci est variable suivant les groupes de génotypes. Mais il est toujours élevé (du $15^{\mathrm{e}}$ au $20^{\mathrm{e}}$ œil audessus de la $1^{\text {re }}$ vrille).

Il est hautement probable que des résultats comparables pourraient être obtenus à partir d'individus développés en tubes. En effet, les phénomènes que nous avons observés concernant le comportement d'individus de «Muscat à petits grains $B$ » et de «Grenache $N$ » issus de multiplications in vitro sont très largement comparables à ceux qui marquent le développement de plantes provenant de graines. 
Dans la mesure où ce type de traitement n'a pas été utilisé, en l'état des connaissances dont nous disposons actuellement, il paraît vraisemblable qu'au cours de la sélection au vignoble, ont été éliminés, sans doute de manière abusive, des individus issus de semences dont le fonctionnement de l'équipement héréditaire présentait un temps de "latence " juvénile assez important pour que les contraintes écologiques et agronomiques (taille, même si

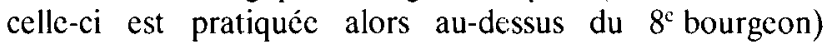
l'empêchent d'accéder à un fonctionnement adulte. En observant le comportement des individus obtenus in vitro pour des variétés telles que le « Muscat à petits grains B. », le "Cinsaut $N$. », le "Grenache $N$. " et, dans la mesure où celui-ci est ainsi que nous l'avons dit, comparable à celui de la plante issue de graine, on peut se demander si ces cépages auraient jamais été sélectionnés dans les conditions agronomiques actuclles. Peut-être tout simplement a-t-on parfois laissé de côté le fait que la vigne est une liane et que l'utilisation de ces pratiques, dont on sait qu'elles sont rajeunissantes, ne permettait pas à ce végétal, au moins pour certains clones, de parvenir à l'état adulte.

\section{CONCLUSION}

De l'ensemble de ces observations, il ressort que, puisque la morphogenèse apparemment inhabituelle obtenuc au vignoble chez des individus issus de culture in vitro n'est pas définitivement fixćc, elle correspond à un des types de fonctionnement du matériel héréditaire de la plante et non à une modification de ce dernier. Aussi bien, ne peut-on pas envisager une explication de ces morphogenèses observécs sur des individus issus soit de graines, soit de plantes miniaturisées cultivées in vitro, comme des mutations (Branas, 1954, 1974; Truel \& Valat, 1971). Une telle explication de ces cas est d'autant moins plausible que ce sont les mêmes modifications qui apparaissent toujours.

De même, peut-on exclure une interprétation faisant intervenir un « débobinage » d'une structure en chimère, ce qui impliquerait que le bouturage in vitro déterminerait dans tous les cas l'intervention de ce phénomène. Ceci semble d'autant moins vraisemblable que, pour de nombreuses autres plantes, l'expérience a montré que ce mode de multiplication conduit à une descendance " conforme " à l'individu de départ.

Les observations rapportées, concordantes tant pour les plantes issues.de semences que pour celles issues de cultures in vitro, paraissent bien être plutôt justiciables d'explications faisant intervenir des modalités diverses de fonctionnement du matériel héréditaire, caractérisant des étapes plus ou moins avancées de la morphogenèse à partir de la phase de fonctionnement juvénile ; celle-ci peut être plus ou moins longuement prolongée par suite de la culture in vitro et perpétuée du fait de contraintes écologiques (peut-être la culture in vitro, sûrement le climat) associées à des pratiques agronomiques particulières (taille).

Ces conclusions, nous semble-t-il, apportent des éléments dont on pourra tenir compte tant sur le plan fondamental que dans la pratique agronomique pour une meilleure gestion de $V$. vinifera.

Reçu le 24 mai 1982. Accepté le 9 mars 1983.

\section{RÉFÉRENCES BIBLIOGRAPHIQUES}

Branas J., 1954. La multiplication végétative comme cause de dégénération. (VII Congrès International de la Vigne et du Vin). Bull. O.I.V., 278, 75-88.

Branas J., 1974. Sélection phytosanitaire. Résultats obtenus jusqu'à ce jour en viticulture et en œnologie par le moyen de la sćlcction visuclle et de la méthode dite de "thermothérapie ". Bull. O.I.V., 519, 370-378

Galzy R., 1961. Confirmation de la naturc virale du court-nouć de la vigne par des essais de thermothérapic sur des cultures in vitro. C.R. Acad. Sci., 253, 706-708.

Grenan S., 1982. Quelques réflexions à propos de modifications morphogénétiques consécutives à la culture in vitro chez la Vigne (Vitis vinifera L.). Ann. Sc. nat. Bot., 4, 135-146.

Grenan S., Truel P., 1983. Réflexions sur un aspect de la variabilité constatéc au cours de la multiplication de variétés de vigne issues de semis. Agronomie, 3 (7).

Huglin P., Julliard B., 1964. Sur l'obtention de semis de vigne très vigoureux à mise à fruits rapide et ses répercussions sur l'amélioration génétique de la Vigne. Ann. Amélior. Plantes, 14, 229-244.

Nozeran R., 1978. Réflcxions sur les enchaînements de fonctionnements au cours du cycle des végétaux supérieurs. Bull. Soc. Bot. Fr., 125, 263-280.

Nozeran R., 1980. La multiplication végétative chez les végétaux supérieurs. Gauthier-Villars, Bordas, Paris, 1-29.

Nozeran R., Bancilhon L., 1972. Les cultures in vitro en tant que techniques pour l'approche de problèmes posés par l'amélioration des plantes. Ann. Amélior. Plantes, 22 (2), 167-185.
Nozeran R., Bancilhon-Rossignol L., Grenan S., 1977. Nouvelles possibilités d'obtention et de multiplication rapide de cloncs sains de Pomme de terre (Solanum tuberosum L.). C.R. Acad. Sci., 285, 36-40.

Nozeran R., Ducreux G., Rossignol L., 1982. Réflexions sur les problèmes de rajeunissement chez les végétaux. Bull. Soc. Bot. Fr., 129. Lettres botaniques, 2, 107-130.

Truel P., Valat C., 1971. A propos de l'Alphonse Lavallée. Progrès agric. et vitic., 24, 515-523.

Valat C., Mur G., 1976. Thermothérapie du Cardinal Rougc. Progrès agric. et vitic., 6, 200-202.

Valat C., Rives M., 1973. Information and comments on variation induced by thermotherapy. 5th meeting I.C.V.G., Salice Termo (Italic). Riv. Pathol. Vég., 4, 9, 291-293.

Valat C., Grenan S., Auran G., Bonnet A., 1979. Guérison de quelques maladies à virus de la vigne par thermothérapie de plantules cultivées in vitro. Vignes et Vins, 284, 19-22.

Valat C., Grenan S., Auran G., 1981. Thermothérapie in vitro: premières observations sur les aptitudes de quelques varićtés de porte-greffes et de Vitis vinifera traitćcs. Vignes et Vins, 298, 17-23.

Wagner R., Bronner A., 1974. Etude de la fertilité des semis de Vitis vinifera : application à la mise au point d'un test précoce de sélection. Ann. Amélior. Plantes, 24 (2), 145-157.

Watelet-Gonod M. C., Favre J. M., 1981. Miniaturisation et rajeunissement chez Dahlia variabilis (variété Télévision) cultivé in vitro. Ann. Sci. nat. Bot., 13 (2, 3), 51-67. 